\title{
Monitoring and analyzing exoplanetary transits from Argentina
}

\author{
Eduardo Fernández-Lajús ${ }^{1}$, Yamila Miguel ${ }^{1}$, Andrea Fortier ${ }^{2}$ and \\ Romina P. Di Sisto ${ }^{1}$ \\ ${ }^{1}$ Facultad de Ciencias Astronómicas y Geofísicas - UNLP, \\ Instituto de Astrofísica de La Plata - CONICET/UNLP, \\ Paseo del Bosque S/N, La Plata, Pcia. Bs. As., Agentina \\ email: eflajus@f caglp.unlp.edu.ar \\ ${ }^{2}$ Physikalisches Institut, University of Bern, Switzerland
}

\begin{abstract}
Photometric observations of transits can be used to derive physical and orbital parameters of the system, like the planetary and stellar radius, orbital inclination and mean density of the star. Furthermore, monitoring possible periodic variations in transit timing of planets is important, since small changes can be caused by the presence of other planets or moons in the system. On the other hand, long term changes in the transit length can be due to the orbital precession of the planets. For these reasons we started an observational program dedicated to observe transits of known exoplanets with the aim of contributing to a better understanding of these planetary systems. In this work we present our first results obtained using the observational facilities in Argentina including the 2.15 telescope at CASLEO.
\end{abstract}

Keywords. planetary systems, stars: variables: other, techniques: photometric

\section{Introduction}

If a planetary System is oriented in space in such a way that the orbital plane is near the observer's visual, the planet will pass periodically in front of the stellar disk producing what is called a transit. Photometric observations of transits can be used to derive physical and orbital parameters of the system, like the planetary and stellar radius, orbital inclination and mean density of the star. Also it is very important to monitoring possible periodic changes in the O-C plots of planets since small changes of the transit moment can be caused by the presence of other planets or moons in the system. On the other hand, long term changes in the transit length can be due to the orbital precesion of the planets. The parameters of a system with transits should be estimated confronting the observations with numerical methods that model the light curves of transits.

Since the discovery of the first exoplanet, known as 51 Peg b by Mayor and Queloz (1995), the number of planets discovered in other stars has grown rapidly. Today we have $\sim 500$ exoplanets detected of which $\sim 100$ are transiting planets. However, these numbers change really very quickly and the new observations present constantly new paradigms to be explained by theories of formation of planetary Systems.

In order to contribute to the knowledge and better understanding of the great diversity of these exoplanetary systems, we started an observational program dedicated to observe transits of known exoplanets. We present here our fist results obtained using the observational facilities in Argentina including the 2.15 telescope at CASLEO.

\section{Observations}

Image acquisition: Digital CCD images were acquired with the 2.15-m "Jorge Sahade" (JS), f/8.4 Cassegrain, telescope at Complejo Astronómico El Leoncito, Argentina. We 


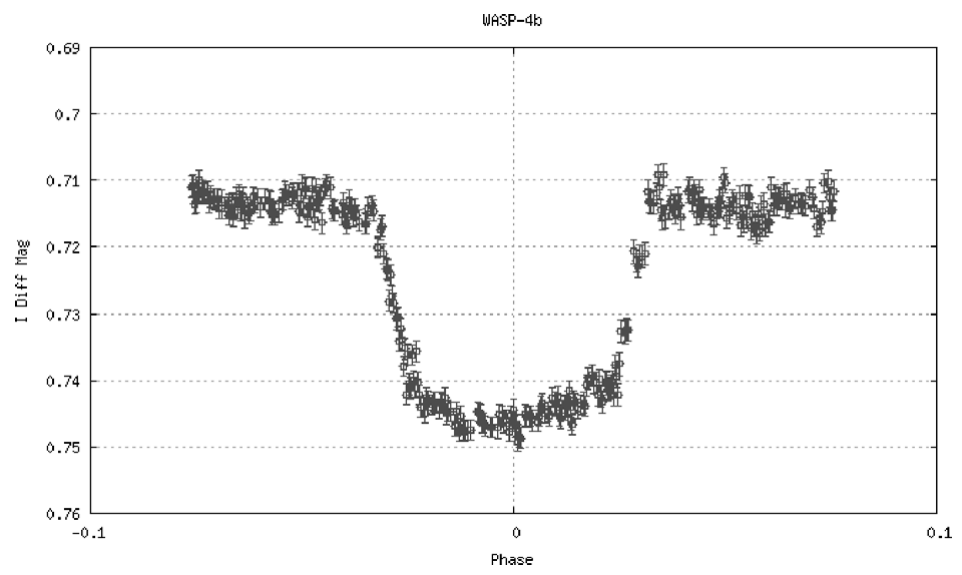

Figure 1. Ligth Curve of WASP-4b.

had two observing runs, with a total of nine usefull nigths during March, 2009 and August, 2010. We used a focal reducer to image a wider field. Image acquisition was performed using a CCD camera attached to the JS telescope, with the broad band filters of the Johnson-Cousins system. The camera is a Versarray 1300B Roper Scientific manufactured by Princeton Instruments. The chip array is $1340 \times 1300$ pixels $(20 \mu \mathrm{m}$ square pixel $)$, the scale being $0.677 " / p x$ (with the focal reducer), with a circular usefull field of $9^{\prime}$. Bias, dark and flat-field frames are acquired every night to calibrate the science images.

Data reduction: Instrumental magnitudes of each star are determined by means of aperture photometry. An image processing pipeline was written in order to practice real-time aperture photometry to the stars contained in the frames. This pipeline was developed making usage of the IRAF command language and some tasks as those of the DAOPHOT photometry package. The aperture radius was selected for each image series in order to maximize the signal-to-noise ratio. Differential magnitudes of the targets are then obtained using an appropriate comparison star in the field and testing the results with some other check stars. Magnitude errors are calculated considering the poisson noise of the source and background, as well as the detector noise and scintillation noise.

\section{Results and conclusions}

During both observing runs we could observe a ten transits either partial or complete. In Fig. 1 we show the ligth curve resulting from our I differential photometry of a planetary transit of Wasp-4b as an example of our detections. Some of our results were already published in the exoplanet transit database (http://var2.astro.cz/ETD/). Our first objective was to test the capability of the telescope and site to detect exoplanetary transits, both the limiting magnitude and the transit depth. We observed targets up to $V \sim 14 \mathrm{mag}$ detecting transit depths up to $\sim 0.01 \mathrm{mag}$. In the coming months and years, we will continue to observe exoplanetary transits in order to obtain parameters of the systems, to detect other perturbing companion planets via the technique of transit timing variations and contribute to the study of exoplanets. We plan to continue using the JS telescope as well as some other facilities in Argentina.

\section{References}

Mayor, M. \& Queloz, D. 1995, Nature, 378, 355 